\title{
Entropy of massive fields near a black hole and vacuum polarization: thermodynamics without statistical mechanics
}

\author{
O. B. Zaslavskii \\ Department of Physics, Kharkov Karazin's National University, Svoboda Sq.4, \\ Kharkov \\ 61077, Ukraine \\ E-mail: aptm@kharkov.ua \\ Starting from the Frolov-Zel'nikov stress-energy tensor of quantum mas- \\ sive fields in the Schwarzschild background, we recover the contribution $S_{q}$ of \\ these field into the entropy of a black hole. For fermions with the spin $s=1 / 2$ \\ $S_{q}>0$, for scalar fields $S_{q}>0$ provided the coupling parameter is restricted \\ to some interval, and $S_{q}<0$ for vector fields. The appearance of negative \\ values of $S_{q}$ is attributed to the fact that in the situation under discussion \\ there are no real quanta to contribute to the entropy, so $S_{q}$ is due to vacuum \\ polarization entirely and has nothing to do with the statistical-mechanical \\ entropy. We also consider the spacetime with an acceleration horizon - the \\ Bertotti-Robison spacetime - and show that $S_{q}=0$ for massive fields similarly \\ to what was proved earlier for massless fields.
}

PACS numbers: 04.70.Dy

\section{INTRODUCTION}

One of the most striking features in black hole physics consists in that a black hole behaves like a thermodynamic system and possesses temperature and entropy. In the state of thermal equilibrium the total entropy $S_{t o t}=S_{b h}+S_{q}$. Here $\mathrm{S}_{b h}=A / 4(\hbar=c=$ 1 ) is the Bekenstein-Hawking entropy ( $A$ is the surface area of the event horizon), $S_{q}$ is the contribution of radiation and matter fields. The presence of quantum fields modifies the entropy and gives rise to a number of nontrivial issues. The most popular one is the 
problem of quantum renormalization of the entropy and removing thermal divergencies due to fluctuations of fields propagating in the vicinity of the horizon (see, e.g. the book [1]). Less attention is paid to the final result of the regularization and its properties, i.e. $S_{q}$ which enters all thermodynamics relations and describes concrete properties of a system. Thermodynamics of a black hole dressed by Hawking radiation was considered in a series of papers [2] but, to the best of my knowledge, for massless fields only.

Some general remarks on possible nontrivial consequences for thermodynamics of a black hole with a strong backreaction of massive fields were suggested in [3] but without discussing the issue of the entropy. It would seem that thermodynamics of massive fields is less interesting than that of massless ones since if a mass of fields is large enough, $m \gg T$, where $T$ is the Hawking temperature, the contribution of thermal excitations into all thermodynamic quantities including entropy, is negligible. For an "ordinary" (without a black hole, say, relativistic star) system this means that all thermal contributions to relevant thermodynamic quantities (energy, free energy, entropy, etc.) are exponentially small. There is, however, a big difference between the behavior of the energy and entropy of such a system. The energy in the limit under consideration tends to nonzero quantity that arises due to effects of vacuum polarization and depends on details of geometry. In contrast to it, the entropy in the limit under consideration remains exponentially small, i.e. negligible.

The situation changes drastically for the case of a black hole. It is essential that the contribution of vacuum polarization and true particles into the stress-energy tensor can

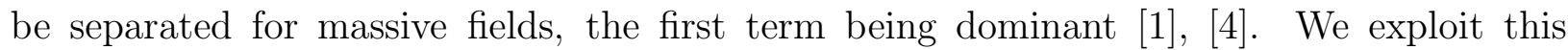
observation for thermodynamics to argue that even in the limit $m \gg T$ we are left with some nonzero contribution into the thermodynamic entropy depending on the geometry of the background and induced by vacuum polarization (zero oscillations) of quantum fields. In fact, in the main approximation the thermodynamic entropy of quantum fields is due to vacuum polarization entirely. Thus, it is the condition $m \gg T$ which reveals the qualitative difference in the thermodynamics of systems with and without black holes. Moreover, it turns out that the contribution under discussion may have any sign (thus, $S_{q}<0$ for the 
$\operatorname{spin} s=1)$.

All what was said above can serve as a motivation to the calculation of $S_{q}$ for massive fields. To solve this problem, we apply, mutatis mutandis, the approach elaborated in previous papers [5], [6] where it was used for the massless case.

\section{ENTROPY IN THE SCHWARZSCHILD BACKGROUND}

Consider a quantum field $\phi$ at finite temperature equal to its Hawking value $T=\beta^{-1}$. Its Euclidean action takes the standard thermodynamic form

$$
I_{q}=-\beta \int d^{3} x \sqrt{g} T_{0}^{0}-S_{q}
$$

Here $T_{\mu}^{\nu}$ is the stress-energy tensor of quantum fields calculated with respect to the background with the metric $g_{\mu \nu}, g$ is determinant of $g_{\mu \nu}$. It is assumed from the very beginning that the renormalization is already performed in $T_{\mu}^{\nu}$, so we may manipulate with $T_{\mu}^{\nu}$ safely. We consider the Schwarzschild background:

$$
d s^{2}=-d t^{2}\left(1-r_{+} / r\right)+\left(1-r_{+} / r\right)^{-1} d r^{2}+r^{2} d \omega^{2}
$$

The system is supposed to be confined in the spherical container of the radius $R$. Consider now the response of the action to the particular class of metric variations caused by the change of the value of $r_{+}$which preserve the general form (2). Then, as was shown in ( [6]),

$$
(4 \pi)^{-2} \frac{\partial I_{q}}{\partial r_{+}}=R^{3} T_{r}^{r}(R)-\int_{r_{+}}^{R} d r r^{2} T_{\mu}^{\mu}
$$

The stress-energy of massive fields for $m \gg T$ has the general structure [4], [1]

$$
\begin{aligned}
T_{\mu}^{\nu} & =\frac{r_{+2}}{r^{8}} f_{\nu}^{\mu}\left(\frac{r_{+}}{r}\right), \\
f_{\nu}^{\mu}\left(\frac{r_{+}}{r}\right) & =\frac{A}{m^{2}} q_{\mu}^{\nu}, q_{\mu}^{\nu}=a_{\mu}^{\nu}+b_{\mu}^{\nu} \frac{r_{+}}{r},
\end{aligned}
$$

where $A, a_{\mu}^{\nu}$ and $b_{\mu}^{\nu}$ are pure numbers.

It is convenient to introduce a variable $u=r_{+} / r, w \leq u \leq 1, w=r_{+} / R$. Then we have the equation 


$$
\frac{\partial J}{\partial w}=w^{2} f_{1}^{1}(w)+w^{-3} \int_{1}^{w} d u u^{4} f_{\mu}^{\mu}(u)
$$

where $J \equiv(4 \pi)^{2} R^{2} I_{q}$. Taking into account the identity

$$
\begin{aligned}
\int_{1}^{w} d u^{\prime} f\left(u^{\prime}\right) \int_{1}^{u^{\prime}} d u^{\prime \prime} g\left(u^{\prime \prime}\right) & =\int_{1}^{w} d u g(u)[\psi(w)-\psi(u)], \\
\frac{d \psi(u)}{d u} & \equiv f(u)
\end{aligned}
$$

and the relationship between the action and entropy (1), we get after simple manipulations:

$$
\begin{aligned}
S_{q} & =\frac{16 \pi^{2}}{m^{2} r_{+}^{2}} A \Phi(w), \\
\Phi(w) & =w^{2} \int_{w}^{1} d u u^{2} \chi_{1}(u)+\int_{w}^{1} d u \chi_{2}(u), \\
\chi_{1} & =q_{1}^{1}+\frac{q_{\mu}^{\mu}}{2}, \chi_{2}=-u^{2}\left(q_{0}^{0}+\frac{q_{\mu}^{\mu}}{2}\right) .
\end{aligned}
$$

The constant of integration is chosen in such a way that $S_{q}=0$ at $w=1, r_{+}=R$ (no room for radiation).

It follows from (7) and from the regularity condition on the horizon $q_{0}^{0}(1)=q_{1}^{1}(1)$, equivalent to $T_{0}^{0}\left(r_{+}\right)=T_{1}^{1}\left(r_{+}\right)$, that $\frac{d \Phi}{d w}{ }_{\mid w=1}=0$, so $\Phi(w)=(1-w)^{2} \rho$, where $\rho$ is some function of $w$, finite at $w=1$. Its explicit form will be given below for different types of fields.

Restoring the usual variables, we can rewrite the contribution of quantum fields in the form

$$
S_{q}=\beta_{H} \int d^{3} x \sqrt{g}\left[\frac{r^{2}}{R^{2}}\left(T_{1}^{1}+\frac{1}{2} T_{\mu}^{\mu}\right)-\left(T_{0}^{0}+\frac{1}{2} T_{\mu}^{\mu}\right)\right]
$$

or

$$
\begin{aligned}
S_{q} & =\int d^{3} x \sqrt{g_{3}} s_{e f}, \\
T_{l o c} s_{e f} & =p+\rho-\left(1-\frac{r^{2}}{R^{2}}\right)\left(\rho+\frac{1}{2} T_{\mu}^{\mu}\right),
\end{aligned}
$$

where $p \equiv T_{1}^{1}$ is the radial pressure, $\rho=-T_{0}^{0}$ is the energy density, $T_{l o c}=T / \sqrt{-g_{o o}}$ is a local Tolman temperature, $g_{3}$ is the determinant of the spatial metric. Eq. (9) replaces the usual thermodynamic Euler relation $T_{l o c} s=P+\rho$. 
The method of the derivation of the expression for the entropy may be applied not only to the quantity $S_{q}$ as a whole but also to the distribution of the entropy in space. It is obvious in our approach that for any layer $r_{+} \leq r \leq r_{0} \leq R$ one obtains the same formulas (घ)-(9) with the replacement $R \rightarrow r_{0}$.

Performing integration in (7) and using the explicit values of the coefficients $a_{\mu}^{\nu}$ and $b_{\mu}^{\nu}$ according to [1] and [4], we obtain after simple calculations (the index indicates the value of the spin of a field):

$$
\begin{aligned}
\Phi_{s} & =(1-w)^{2} \rho_{s}, \\
\rho_{0} & =(122-546 \xi) w^{4}+(94-420 \xi) w^{3}+(66-294 \xi) w^{2}+(38-168 \xi) w+19-84 \xi \\
\rho_{1 / 2} & =8+16 w+30 w^{2}+44 w^{3}+58 w^{4}, \\
\rho_{1} & =-3\left(9+18 w+32 w^{2}+46 w^{3}+60 w^{4}\right) .
\end{aligned}
$$

\section{DISCUSSION}

Let us now discuss the obtained results. In the spirit of ( [2] ) for the scalar field we could derive from the conditions of the absence of layers with the negative entropy $\Phi \geq 0$ and $\frac{d \Phi}{d w}<0$ (equivalent to $\left(\frac{\partial S_{q}}{\partial R}\right)_{r_{+}}>0$ ), the restrictions on the coupling parameter $\xi_{1} \leq \xi \leq \xi_{2}$, where in our case $\xi_{1}=0.2142858$ and $\xi_{2}=0.2242068$. Thus, both the conformal $(\xi=1 / 6)$ and minimal $(\xi=0)$ couplings are outside this range. However, the example with the vector field especially clearly shows that the values $S_{q}<0$ are admissible, so restriction on the coupling parameter in the scalar case can be relaxed at all.

As the result $S_{q}<0$ looks rather surprising, we would like to stress the following. First, the expression for the tensor (4) was derived under the assumption that $m \gg T$. It means that the contribution of thermal excitations to the entropy and other thermodynamic quantities are strongly damped with the exponential factor $\exp (-m / T)$ and one deals with the vacuum polarization effects entirely. In the limit $R \rightarrow \infty T_{\mu}^{\nu} \rightarrow 0$ in contrast to the massless case when the tensor $T_{\mu}^{\nu}$ approaches that of thermal radiation in the flat space. 
The contribution from the statistical-mechanical part $S^{S M}$ (that is the sum over modes with the Bose distribution function) is absent in the main approximation - correspondingly, any reasonable statistical interpretation of $S_{q}$ cannot be given and this abolishes the prohibiting of $\mathrm{S}_{q}<0$.

Second, the quantity $S_{q}$ enters the expression for the total entropy of a black hole with its counterpart $S_{b h}$ only: $S_{t o t}=S_{b h}+S_{q}$. As $S_{q}$, by its very meaning, represents small corrections to $S_{b h}$, it is obvious that $S_{t o t}>0$. The term $S_{q}$ arises due to the presence of a black hole and cannot be "cut" from it and considered on its own. For comparison, in the vicinity of a relativistic star there is no $S_{b h}$, but $S_{q}$ is due to thermal excitations only and is positive (exponentially small, if $m \gg T$ ).

Usually, the contribution to the total thermodynamic entropy of a black hole which comes from quantum fields can be represented in the form

$$
S_{q} \equiv S^{T M}=S^{S M}+S_{0}
$$

where $S^{S M}$ is the statistical-mechanical part of the entropy and $S_{0}$ is some qiantity depending on parameters of the geometry (say, the horizon radius in the Schwarzschild case). Both $S^{S M}$ and $S_{0}$ diverge due to the contribution of the near horizon region but their sum is finite. This scheme explains the general mechanism of the black hole entropy renormalization [1], [7], [8]. The quantity $S^{T M}$ represents the thermodynamic entropy, i.e. the entity that enters the general thermodynamic relations - in particular, it describes the response of the free energy to the change of temperature [9].

The representation (14) was directly justified in the terms of the stress-energy tensor for massless fields [6]. In that case

$$
S_{q}=16 \pi^{2} r_{+} \int_{r_{+}}^{R} d r r^{2}\left(T_{1}^{1}-T_{0}^{0}-T_{\mu}^{\mu} \ln \frac{R}{r}\right)
$$

Using the splitting for the stress-energy tensor of quantum fields in the Hartle-Hawking state [10

$$
T_{\mu}^{\nu}=\left(T_{\mu}^{\nu}\right)_{B}+\left(T_{\mu}^{\nu}\right)_{t h}
$$


(index "B" denote the Boulware state, "th" stands for thermal radiation) we obtain just (14) with $S^{S M}$ identified with the entropy $S^{\text {th }}$ of thermal radiation and $S_{0}$ obtained by the replacement of $T_{\mu}^{\nu}$ in (15) by $\left(T_{\mu}^{\nu}\right)_{B}$.

For the case of massive fields the situation is quite different. Now $S^{S M}=0$ in the main approximation with respect to the parameter $T / m$, while $S_{0}$ is finite and should be identified (up to small corrections) with $S_{q}$.

The fact that $S^{S M}$ is discarded has important consequences for the scheme of a black hole renormalization in the case of massive fields. The detailed discussion of this issues is beyond the scope of the present paper, so we will restrict ourselves by several remarks. In the paper [11] it was observed that using Pauli-Villars regularization enables one to obtain the finite value of the entropy without introducing a cutoff parameter typical for the brick wall model. The approach of [1] well explains the mechanism of cancelling divergencies but, in our view, cannot give the correct value for the finite part of $S_{q}$ describing the contribution of matter fields that depends on the position of the shell surrounding a black hole. (And, in fact, the authors of [11] did not pose the purpose of full calculating such a quantity and themselves emphasize that they neglect contributions to the entropy that does not diverge as the mass of the regulator fields tends to infinity. Meanwhile, this contribution is essential for calculating the entropy contained between the horizon and the shell, i.e. the quantity we are seeking for.) This is due to the fact that $S_{q}$ is represented in [11] as the linear combination of quantities $S^{S M}$ for different kinds of fields, while the contribution of corresponding terms $S_{0}$ was not taken into account. Meanwhile, as we emphasize, $S^{S M}=0$ in the main approximation at hand, the main contribution to $S_{q}$ comes from the vacuum polarization, so account for $S_{0}$ (not made in [11]) is crucial for our task.

It is worth stressing that, although $S_{q}$ cannot be given statistical interpretation, it does contribute to thermodynamic relations (for instance, into the general first law), so we have "thermodynamics without statistical mechanics". As it is statistical interpretation that demands $S_{q}>0$, the lack of such an interpretation opens the possibility for $S_{q}<0$. 


\section{BERTOTTI-ROBINSON SPACETIME}

Discussion above refers to the black hole background. Now we will consider another type of spacetime with a horizon - Bertotti-Robinson spacetime (BR) that, as is known, possesses the acceleration horizon [12], [13]. Recently it was shown that in such a spacetime $S_{q}=0$ for massless fields [14] (generalization to other spacetimes with an acceleration horizon was suggested in [15]). This fact was attributed to the pure kinematic nature of an acceleration horizon which does not produce "true" quanta. This suggests that the result $S_{q}=0$ must hold true for massive fields on the same footing as in the massless case. Below we confirm it by direct calculation.

The variation of the action with respect to the metric in the BR has one subtlety: as the radius $r_{+}$enters the expression for the angular part of the metric on a boundary, the components $g_{\theta \theta}$ and $g_{\phi \phi}$ vary along with components $g_{00}$ and $g_{r r}$. In particular, in the formula for the first general law this gives rise to additional terms responsible for the gravitational contribution of the pressure. These points are discussed in details in [15], so below we only list the corresponding results without derivation.

The metric under discussion has the form

$$
\begin{aligned}
d s^{2} & =-d t^{2} b^{2}+d l^{2}+r_{+}^{2} d \omega^{2}, \\
b & =r_{+} d(z), d=\operatorname{sh} z, z=l / r_{+} .
\end{aligned}
$$

In this background the stress-energy of massive fields reads [16], 17]

$$
T_{\mu}^{\nu}=\frac{C}{r_{+}^{6}}(1,1,-1,-1) .
$$

Here $C=c / m^{2}, c$ is a numerical constant whose exact value is irrelevant for our purposes. From dimensional arguments and the structure of $T_{\mu}^{\nu}$ it follows that the semiclassical action of a massive quantum field confined in the region $0 \leq z \leq z_{B}$

$$
I_{q}=\frac{J\left(z_{B}\right)}{r_{+}^{2}}
$$

Now let us take advantage of the relation (see eq. (10) of 15): 


$$
\left(\frac{\partial I}{\partial z}\right)_{r_{B}}=-8 \pi^{2} r_{+}^{2} d(z) T_{1}^{1}
$$

Then we obtain that

$$
J=-8 \pi C \int_{0}^{z_{B}} d z^{\prime} d\left(z^{\prime}\right)
$$

where we took into account that $J=0$ if $z_{B}=0$ (no room for quantum fields). Comparing (19), (21) with (11), we get immediately $S_{q}=0$.

This result coincides with that in [14], [15] and can be explained by the same reason. It consists in that the horizon of the BR is not a true black hole horizon but represents a pure kinematic effect, too weak to gain nonzero entropy. However, there is also a difference between interpretation of the property $S_{q}=0$ for massless and massive fields. In the first case this property can be thought of as the result of the mutual compensation between two divergent contributions $\left(S^{S M}\right.$ and $\left.S_{0}\right)$ from thermal excitations and vacuum polarization. For the massive field case the first contribution is absent, so we have $S_{0}=0$.

\section{CONCLUSION}

To the best of my knowledge, the situation we discussed is the first example when the thermodynamic entropy arises due to vacuum polarization effects, so the entropy reveals itself even in spite of the absence of thermal excitations. This phenomenon is due to the presence of an event horizon and is absent, for instance, in the background of a relativistic star. In the latter case quantum-gravitational polarization effects cannot themselves produce entropy that may arise due to thermal excitations of quantum fields only.

The unusual situation, called above "thermodynamics without statistical mechanics", reveals itself, in particular, in that values of corrections $S_{q}<0$ are allowed. In spite of the difference between two examples considered in the present paper they share the common feature - the absence of "true" quanta that resulted in the unusual properties $S_{q}<0$ (Schwarzschild background) and $S_{q}=0(\mathrm{BR})$. It would be of interest to verify the properties outlined above in other black hole spacetimes. 
In conclusion, let me point out the important issues that remained beyond the scope of the present paper and need separate treatment. It would be interesting to present microscopic description of results obtained and understand, how they can be interpreted in terms of counting quantum states. This problem becomes especially interesting in the context of recent developments in the framework of the D-brane approach.

\section{ACKNOWLEDGMENT}

I thank H. Nicolai and Albert Einstein Institute, where this work was performed, for hospitality. I am grateful to S. N. Solodukhin for reading the manuscript and discussion.

[1] V.P. Frolov and I.D. Novikov, Black Hole Physics (Kluwer Academic Publishers, vol. 1996, Chapter 11.1.3.7).

[2] D. Hochberg, T.W. Kephart and J.W. York, Jr, Phys. Rev. D 48 (1993) 479.

P.R. Anderson, W.A. Hiscock, J. Whitesell and J.W. York, Jr, Phys. Rev. D 50 (1994) 6427.

D. Hochberg and S.V. Sushkov, Phys. Rev. D 53 (1996) 7094.

[3] O.B. Zaslavskii, Class. Quant. Grav. 8 (1991) L141-L145.

[4] V.P. Frolov and A.I. Zel'nikov, Phys. Lett. B 115 (1982) 372.

[5] O.B. Zaslavskii, Phys. Lett. A 181 (1993 )105.

[6] O.B. Zaslavskii, Class. Quant. Grav. 13 (1996 ) L23.

[7] V.P. Frolov Phys. Rev. Lett. 74 (1995 ) 3319.

[8] W.H. Zurek and K.S. Thorne, Phys. Rev. Lett. 54 (1985) 2171.

[9] For non-minimaly coupled matter the mechanism of renormalization of the black hole entropy 
has some additional subtleties (see S. N. Solodukhin, Phys. Rev. D 56 (1997) 4968) but we do not discuss this issue here.

[10] S. M. Cristensen and S.A. Fulling, Phys. Rev. D 15 (1987) 2089; P.Candelas, Phys. Rev. D 21 (1980) 2185; V.P. Frolov and K.S. Thorne, Phys. Rev. D 39 (1989) 2125.

[11] J-G. Demers, R. Lafrance and R. Myers, Phys. Rev. D 52 (1995) 2245.

[12] Robinson I Bull.Acad.Pol.Sci. 7 (1959) 351.

Bertotti B Phys. Rev. 116 (1959 ) 1331.

[13] B. Carter General theory of stationary black hole states. In: Black Holes, edited by De Witt C and De Witt B S (New York: Gordon and Breach, 1973).

[14] O.B. Zaslavskii, Phys. Rev. D 57 (1998 ) 6265.

[15] O.B. Zaslavskii, Class. Quant. Grav. 17 (2000) 497-512.

[16] L.A. Kofman and V. Sahni, Phys. Lett. B 127 (1983) 197.

[17] J. Matyjasek, Phys. Rev. D 61, 124019 (2000). 